\title{
PERIODIC BOUNDARY CONDITIONS IN THE PARALLEL EIGENSOLVER OM3P: APPLICATION OF THE INEXACT LANCZOS PROCESS TO HERMITIAN SYSTEMS*
}

\author{
J. F. DeFord and B. Held, Simulation Technology \& Applied Research, Inc., Mequon, WI, USA
}

\section{Abstract}

This paper details the development of periodic boundary condition support in the parallel finite-element eigensolver OM3P. OM3P is based on the inexact Lanczos/JOCC hybrid method, and it uses the vectorscalar (A-V) basis set that has previously been shown to dramatically reduce the computational requirements of the method. We will discuss the implementation challenges, including proper handling of problem partitioning/load balancing for periodic problems. The use of A-V basis introduces additional difficulties in the parallel domain, and we will show solutions to these problems as well omega-beta diagrams for the RDDS structure generated on a modest computer cluster using the software.

\section{THE INEXACT LANCZOS METHOD}

The shift-invert Lanczos method is a standard technique for solving the generalized eigenvalue problem

$$
A x=\lambda B x
$$

when the coefficient matrices are large and sparse, such as arise in eigenmode analysis of rf cavities using the finiteelement method. In the standard approach, a shifted linear system is formed to facilitate the rapid convergence of eigenvalues near the shift point,

$$
(A-\sigma B)^{-1} B x=\frac{1}{\lambda-\sigma} x
$$

and at each Lanczos step the shifted system $A-\sigma B$ must be solved to high accuracy. The shifted system is often highly ill-conditioned, leading to very poor convergence of iterative solvers such as conjugate gradient that are used for large systems that cannot be factored. Consequently, solution times can be unacceptably long even for relatively small problems.

The coupling of inexact solutions to the shifted linear system (Inexact Shift-Invert Lanczos - ISIL) followed by Jacobi Orthogonal Component Correction (JOCC) steps was proposed by Y. Sun, et al.[1], to address this problem. The method was first implemented in the Omega3P code developed at Stanford Linear Accelerator Center, and later in the OM3P solver developed by Simulation Technology $\&$ Applied Research [2]. The technique has been shown to be much faster than the traditional Lanczos process for large systems, is highly efficient on parallel computers, and has been further developed by the original authors for complex symmetric (lossy) systems. In this paper we discuss an additional extension to Hermitian systems that arise from the application of periodic boundary conditions.

\section{PERIODIC BOUNDARY CONDITIONS}

The need for periodic boundary conditions arises in the design of rf beamline cavities, where it is of interest to know the resonant behavior of a periodic (or approximately periodic) system of cavities with a specified phase advance per cell. When the phase advance is a rational fraction of 360 degrees, one can in principle obtain the desired mode by including enough cavities in the analysis. A much more computationally efficient approach is to model one cavity but enforce the requirement that the fields on the entrance and exit planes (periodic boundaries) differ only by the desired phase advance, i.e.,

$$
\left.\vec{E}\right|_{\text {plane 1 }}=\left.e^{-j \phi} \vec{E}\right|_{\text {plane 2 }}
$$

If the matrix equation obtained via the finite element method without periodic boundaries is given by (1), then the application of periodic boundaries transforms the equation as follows:

$$
P^{T} A P x^{p}=\lambda P^{T} B P x^{p}
$$

where superscript " $T$ " indicates conjugate transpose, $x^{p}$ is the subset of unknowns that excludes those that occur on one of the periodic planes (referred to as "dependent" unknowns/sources). Assuming the volume unknowns are enumerated first, followed by those on the independent periodic plane, followed by those on the dependent plane, the matrix $P$ is given by

$$
P=\left[\begin{array}{cc}
I_{n} & 0 \\
0 & I_{m} \\
0 & e^{-j \phi} I_{m}
\end{array}\right]
$$

where $n+m$ is the total unknown count, and $m$ is the number of unknowns on the independent periodic plane. $I_{m}$ is the identity matrix of order $\mathrm{m}$, and $\phi$ is the phase advance across the cell.

\section{APPLICATION TO A-V FORMULATION}

In finite-elements used for electromagnetics the basis set is composed of vector functions tied to specific element entities, including edges, faces, and cells (depending on the order of interpolation). Recent work [3] has shown that the addition of scalar functions that are tied to the nodes yields substantial improvements in the convergence rate of iterative solvers such as conjugate 
gradient, and this approach has been implemented in OM3P. The resulting matrix equation can be written in the form

$$
G^{T} A G x=\lambda G^{T} B G y
$$

where $G$ is very sparse (at most 3 entries per row). Converting to the periodic case yields,

$$
P^{T} G^{T} A G P x=\lambda P^{T} G^{T} B G P y
$$

By eliminating the scalar unknowns on the periodic faces, we can interchange the order of $G$ and $P$ in Eqn. 7, which allows us to express the shifted form as

$$
G_{p}^{T} B_{p} G_{p} x^{p}=\frac{1}{\lambda-\sigma} G_{p}^{T}\left(A_{p}-\sigma B_{p}\right) G_{p} x^{p}
$$

where

$$
A_{p}=P^{T} A P \quad B_{p}=P^{T} B P
$$

and the rows of $G$ that correspond to unknowns on the dependent periodic surface have been removed to form $G_{p}$. To improve efficiency in OM3P the $A_{p}$ and $B_{p}$ matrices are formed explicitly.

\section{PERIODIC PARTITIONING}

To run a problem on a parallel computer, the mesh must be partitioned into parts that can be analyzed on each process. An efficient partition is one that minimizes the amount of communication between processes as compared to the amount of computational work each process must perform. For this reason, good partitioners tend to create mesh partitions that are blocks of contiguous elements. This is not necessarily appropriate for periodic problems because the periodic planes are logically connected, even though they are physically separated in the mesh.

Fig. 1 shows the procedure used in OM3P for partitioning periodic problems. The mesh is initially distributed by sending an equal number of nodes to each process, and then sending full element support for each node set, i.e., a given process receives all elements that connect to any of the nodes that are owned by the process.

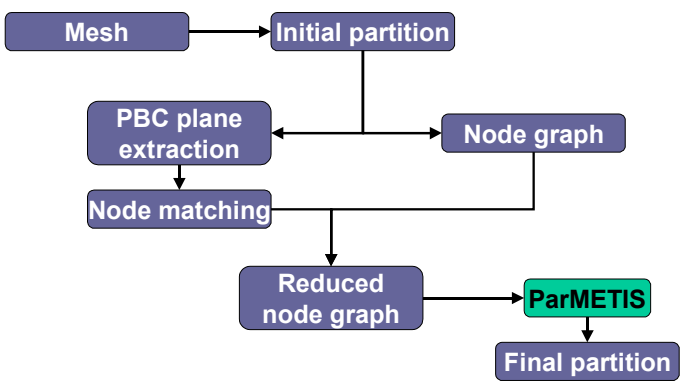

Fig. 1: Periodic partitioning flowchart.

A node graph is created from the initial partition, which is a list of nodes connected to each node via an edge.
Nodes on the independent periodic plane are then matched with the corresponding nodes on the dependent plane, and this information is used to reduce the node graph to one that excludes the nodes on the dependent surface (nodes connected to dependent nodes are connected to the corresponding independent nodes in the reduced graph). The reduced graph is used by ParMETIS [4] to create a load-balanced partition in which independent-dependent node pairs are always found on the same process. In OM3P, all of these steps (after the initial partition) occur on the distributed mesh because we assume that no one process can hold the entire mesh even for pre-assembly processing. Thus, even matching of nodes between dependent and independent planes requires inter-process communication.

A comparison of the results of this partitioning procedure as compared to the non-periodic case is illustrated in Fig. 2.

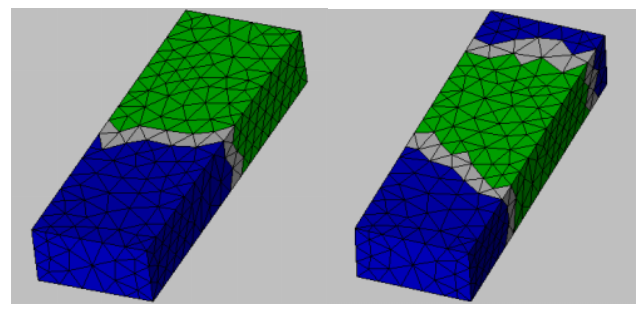

Fig. 2: Two-processor partitions for non-periodic case (left), and periodic case (right). Periodic boundary conditions were applied on the small faces on both ends of the model.

\section{COMPUTATIONAL RESULTS}

OM3P has been applied to a variety of periodic structures, with one example shown in Figs. 3-4. The ISIL-JOCC procedure works well for periodic problems formulated with the A-V basis, and we have been able to run problems with in excess of $400 \mathrm{~K}$ elements on dual processor high-end workstations in a few minutes of cpu time. The floating-point operation count is greater by a roughly a factor of two for the periodic case because the solution vectors are now complex (the matrix is still mostly real, and since the real and imaginary parts are stored separately we incur only a small increase in storage requirements for the coefficient matrix). A modest decrease in convergence rate of the iterative solver has been observed as compared to similar cases where periodic boundaries are not present (for instance, comparing the non-periodic case to the zero phase advance case). This behavior is attributed to the elimination of scalar unknowns on the periodic faces.

\section{CONCLUSIONS}

Support for periodic boundary conditions has been implemented in OM3P. Testing indicates no significant problems in using ISIL-JOCC method on the resulting 
Hermitian matrices. Some degradation in performance is seen due to elimination of scalar unknowns on periodic surfaces, and future work will re-examine this requirement.

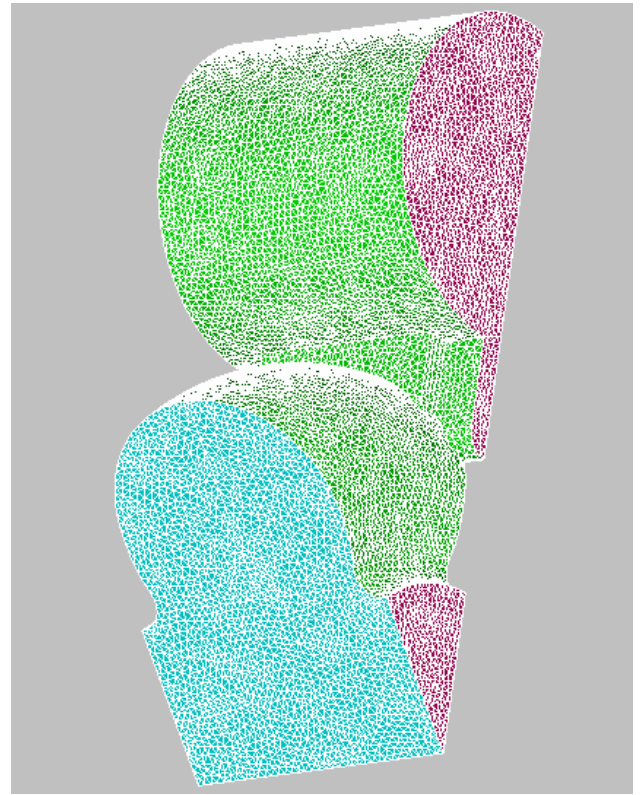

Fig. 3: 1/8 model of RDDS structure (one cell axial width). Periodic boundary conditions applied to axial faces. Approximately $400 \mathrm{~K}$ elements.

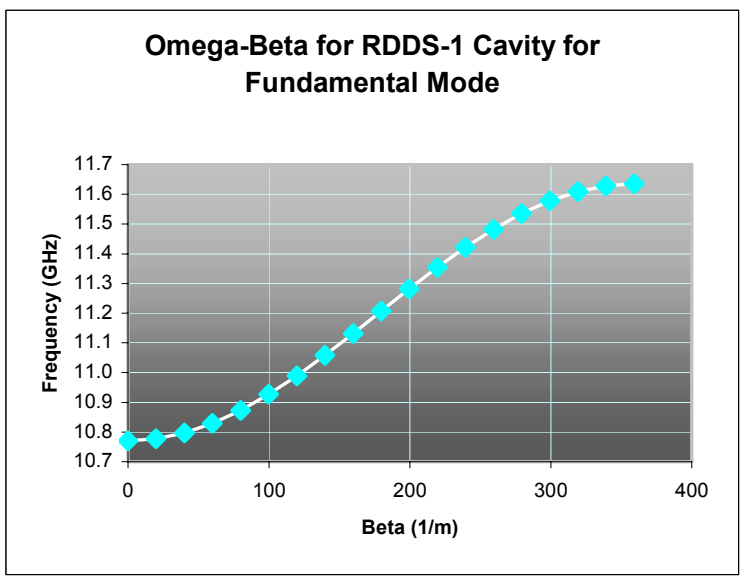

Fig. 4: Dispersion diagram for RDDS cavity.

\section{REFERENCES}

[1] Y. Sun, N. Folwell, Z. Li, and G. Golub, "High precision accelerator cavity design using the parallel eigensolver Omega3P," Proceedings of The Annual Review of Progress in Applied Computational Electromagnetics 2002.

[2] J. F. DeFord and B. Held, "Enhancements to iterative inexact Lanczos for solving computationally large finite element eigenmode problems," Proceedings of the 2002 International Conference on Computational Accelerator Physics, Oct. 15-18, 2002, Michigan State University, East Lansing, MI.

[3] R. Dyczij-Edlinger, G. Peng, and J. F. Lee, "A fast vector-potential method using tangentially continuous vector finite elements," IEEE-MTT, 46, June, 1998, pp.863-868.

[4] ParMETIS is a parallel, graph-theory based, unstructured grid partitioner developed/maintained by G. Karypis, et al., at the University of Minnesota. 